\title{
Structural studies of Cysteine Synthase Complex obtained from Klebsiella pneumoniae
}

\author{
Shubham Semwal ${ }^{1}$, Deepansh Mody ${ }^{2}$, Vibha Gupta $^{2}$, Julie Bouckaert ${ }^{1}$ \\ ${ }^{1}$ Unité de Glycobiologie Structurale et Fonctionnelle, UMR 8576 from the CNRS and the University of Lille, 50 Avenue Halley, \\ 59650 Villeneuve d'Ascq, France, \\ ${ }^{2}$ Biotechnology Department, Jaypee Institute of Information Technology (JIIT), A-10, sector-62, Noida, 201309, India \\ shubham.semwal@univ-lille.fr
}

De novo cysteine biosynthesis is a pathway responsible for metabolizing inorganic sulfur to produce L-cysteine, that holds significance in cellular activities of several organisms, majorly plants and microbes. Absence of this pathway in humans, accompanied by differential roles of accumulated L-cysteine in aiding adaptation of microbes in harsh host environment, promoting toxin inactivation, biofilm formation, and development of antimicrobial resistance (AMR), make this pathway a lucrative target for novel therapeutics. The pathway is fuelled by a bienzyme complex, the Cysteine Synthase Complex (CSC), made up of CysE and CysK enzymes, where a hexameric CysE binds two CysK dimers, one on either end, to further cysteine production in a two-step fashion from L-serine. Recognition of industrial value in addition of the therapeutic potential of this pathway urges the need for further molecular investigations for its optimal exploitation. However, the large size of the molecular complex has put to question the feasibility of resolving its 3-D structure, partially reasoning the inability to obtain a 3-D structure even after nearly three decades of the complex's discovery. Klebsiella pneumoniae, a pathogen with its multi-drug resistance (MDR) recognized in WHO's list of six extensively MDR pathogens grouped as ES ' $K$ ' APE group of pathogens. Targeting the CSC of $K$. pneumoniae is a promising approach to up our armamentarium against the growing menace caused by the pathogen.

The success of rational structure-based drug discovery is driven by the availability of a crystal structure of the target protein complex. With a recent study reporting the SAXS-modelled CSC from E. coli, we aim to model the CSC complex using the K. pneumoniae CysE structure previously made available by our group, adopting a knowledge-based approach. Present poster reports the purification and characterization of recombinant CSC from K. pneumoniae, as an essential first step in progressing towards understanding its structure and biochemical function. Purified recombinant CSC was obtained and subjected to - (i) gel filtration chromatography to separate and estimate molar mass of the complex, (ii) dynamic light scattering for determining hydrodynamic radius and verifying homogenous population, and (iii) negative stain electron microscopy to visualize the purified complex. Simultaneous utilization of in silico tools integrated with techniques of structural biology is being carried to predict the 3-D structure of CSC, and identify residues involved in protein-protein interface stabilization. Following physical analysis and attempts to crystallize the CSC complex obtained from K. pneumoniae we observed heterogeneity in the CSC preparation, with a flexible/dynamic nature of the association between the two interacting proteins posing considerable challenges. Additionally, identification of interacting residues using in silico predictions would facilitate better understanding of useful molecular recognition inferences within CSC, which presently are far from being wellunderstood.

Keywords: de novo cysteine biosynthesis; Cysteine Synthase Complex; Electron Microscopy; Klebsiella pneumoniae; protein-protein interactions; knowledge-based modelling. 